\title{
Maintaining sub-pixel alignment for a single-camera two-bucket shearing speckle interferometer
}

\author{
Peter A A M Somers and Nandini Bhattacharya \\ Optics Research Group, Delft University of Technology, Lorentzweg 1, NL-2628 CJ Delft, \\ The Netherlands \\ E-mail: P.A.A.M.Somers@TNW.TUDelft.nl and N.Bhattacharya@TNW.TUDelft.nl
}

Received 8 November 2004, accepted for publication 11 January 2005

Published 12 May 2005

Online at stacks.iop.org/JOptA/7/S385

\begin{abstract}
In a phase stepped speckle interferometer, phase steps can be realized temporally or spatially. The spatial approach has the advantage of simultaneous acquisition of all phase stepped interferograms which eliminates phase errors due to phase changes that can occur between phase steps when phase is stepped in time. For spatially phase stepped systems based on multiple optical channels, it is important that interferograms are well aligned to the others. Alignment can be achieved and maintained by a system that does not rely strongly on high mechanical stability but is based on measuring the alignment errors and correcting them. The alignment system presented in this work uses speckle correlation to quantify alignment errors with sub-pixel accuracy, and comprises a piezo-electric controlled mirror to achieve precise alignment.

The alignment method has been implemented for a two-channel shearing speckle interferometer. It allows for precise initial alignment both for translation and rotation of the two phase stepped speckle patterns on the CCD. Translation adjustments to maintain sub-pixel alignment during operation can be realized very quickly, preceding a test session. The importance of adequate alignment, and the effects of insufficient alignment, are illustrated by experimental results.
\end{abstract}

Keywords: speckle interferometry, shearography, alignment, speckle correlation

(Some figures in this article are in colour only in the electronic version)

\section{Introduction}

In a phase stepped speckle interferometer, phase steps can be realized temporally [1] or spatially [2]. Temporal phase stepping has the advantage that all phase stepped interferograms are projected onto a single camera after passing the same optical system. This makes such systems less sensitive for geometrical aberrations of the optics. An arbitrary number of phase stepped interferograms can be taken for increased error tolerance. However, phase changes can occur between phase steps when phase is stepped in time, resulting in phase errors.

Spatial phase stepping interferometers can be categorized into single-channel and multi-channel systems. In single- channel systems a spatial frequency carrier is generated by tilting the reference beam. The phase distribution can then be calculated by the Fourier method [2]. In a particular implementation the tilt is chosen such that the phase difference between adjacent pixels is $\pi / 2$ or $2 \pi / 3$, allowing for the calculation of phase in the space domain by well known phase stepping algorithms [3].

The alternative to spatial phase stepping in a single image is the use of multiple phase stepped channels. Separation and phase stepping of interferograms can be implemented by fixed diffraction gratings [4-6] or by computer-generated diffractive elements [7]. Another approach for separating and phase stepping the interferograms is based on custom 


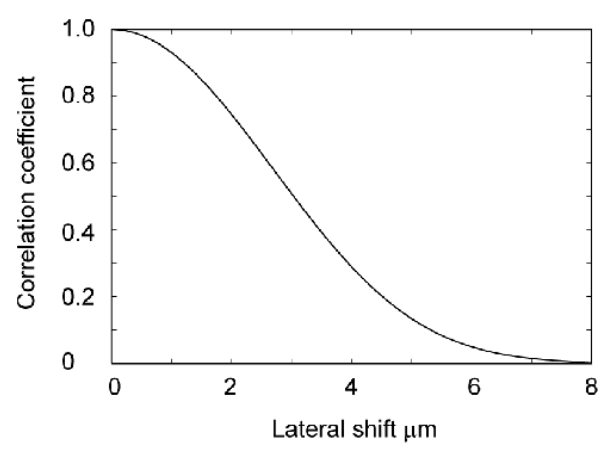

(a)

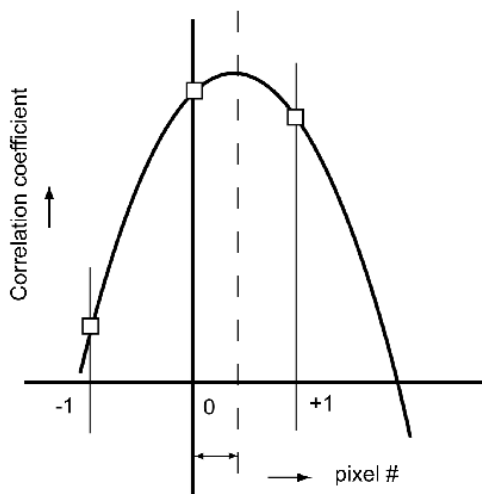

(b)

Figure 1. (a) Correlation between two laterally shifted speckle patterns. (b) Three-point parabola fitting of correlation coefficients to obtain sub-pixel resolution.

made polarization sensitive elements [8], Savart elements [9], or more conventional components involving polarizing beam splitters and wave plates, as has been the choice for the present interferometer [10].

The spatial approach has the advantage of simultaneous acquisition of all phase stepped interferograms, which eliminates errors caused by changes of the object or the environment during temporal phase stepping. However, when spatial phase stepping is implemented in a multi-channel system, care has to be taken that phase stepped interferograms remain well aligned. This is a non-trivial task: assuming that speckle size is of the order of pixel size, each interferogram has to be aligned with sub-pixel accuracy to the other phase stepped interferograms.

There are three issues that have to be considered when combining phase stepped speckle interferograms obtained in a multi-channel optical system. The most obvious is translation alignment in two directions: $x$ - and $y$-coordinates of all interferograms involved must match. Another source of error is rotation of an image compared to the other(s). This can be caused by tilt errors in $45^{\circ}$ mirror surfaces that are present in the optical system. A final category concerns geometrical differences between images, that may be caused by different focusing, magnification or distortion by the imaging optics. In a $1024 \times 1024 \mathrm{CCD}$ camera a difference of $0.1 \%$ between channels already leads to an error of one pixel!

Alignment with respect to position and orientation of phase stepped interferograms might be achieved and maintained by employing a mechanical design that is highly stable, under all ambient conditions, which is a difficult task, and results in an expensive and heavy system. We have chosen an alternative that does not rely strongly on high mechanical stability but is based on measuring the alignment errors and correcting them. The alignment system uses speckle correlation to quantify alignment errors between two phase stepped speckle interferograms with sub-pixel accuracy, and comprises a piezo-electric controlled mirror to achieve precise alignment.

\section{Methods}

Speckle patterns contain high spatial frequencies when speckle size is of the order of pixel size, as is the case in our speckle interferometer. Correlation methods are sensitive for high frequency content and therefore are a good candidate for quantifying shifts between two identical speckle patterns. Quantification of speckle displacement by correlation methods was pioneered by Yamaguchi [12] and has seen extensive use in strain analysis since then, both in research [13] and in commercial instruments, replacing earlier methods, in particular speckle photography. These methods produce $2 \mathrm{D}$ resolved results and are based on correlation between a reference speckle pattern acquired before a displacement and a shifted one, taken thereafter. In general, these speckle patterns are very similar to each other, at least locally.

Our phase stepped speckle patterns are not identical, due to modulation that causes intensity differences for many corresponding pixels in the phase stepped speckle patterns, in particular when one of the interfering beams is uniform. For speckle interferometers that use a speckle reference beam, such as shearography, there is no modulation on many pixel positions, due to the presence of a 'dark' speckle in one of the interfering fields. In the other field a speckle with nonzero intensity may be present, and at these locations the phase stepped interferograms are identical, which contributes to a non-zero correlation coefficient when correlating the speckle patterns. Although it is possible to use partly modulated speckle patterns for alignment purposes, it is preferred to prevent modulation for a more reliable procedure. Correlation between two speckle patterns $k$ and $l$ can be calculated by [11]

$$
c_{k l}=\frac{\left\langle I_{k} I_{l}\right\rangle-\left\langle I_{k}\right\rangle\left\langle I_{l}\right\rangle}{\sqrt{\left\langle\left(I_{k}-\left\langle I_{k}\right\rangle\right)^{2}\right\rangle\left\langle\left(I_{l}-\left\langle I_{l}\right\rangle\right)^{2}\right\rangle}},
$$

where $I_{k}$ and $I_{l}$ are the $x, y$-dependent intensities of patterns $k$ and $l$ respectively.

An example of the use of equation (1) is given in figure 1(a). It shows the correlation coefficient for a simulated speckle intensity distribution, correlated with itself in different shift positions. The speckle pattern was modelled by a simple ray tracing model: the contribution of 100 randomly chosen points on a $0.1 \times 0.1 \mathrm{~m}$ object, with random phase, was coherently added at each point in a $100 \mu \mathrm{m} \times 100 \mu \mathrm{m}$ observation plane, using a $1 \mu \mathrm{m}$ grid. The distance between the object and the observation plane was $0.6 \mathrm{~m}$. Average speckle size was $3 \mu \mathrm{m}$, using a wavelength of $1 \mu \mathrm{m}$. The correlation 


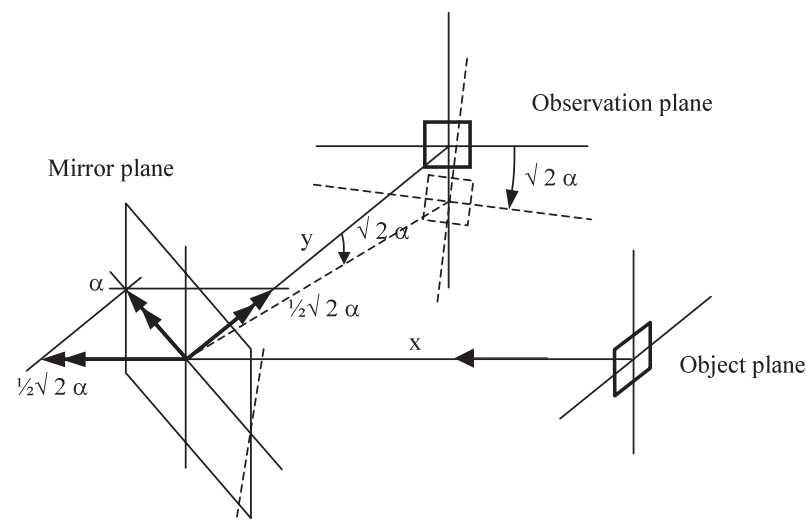

Figure 2. Rotation of an image by a $45^{\circ}$ mirror, rotated over its horizontal axis, decomposed over the $x$ - and $y$-axes.

coefficient decays like a Gaussian distribution as a function of speckle pattern shift and might be represented by a simple polynomial for small shifts.

The $x$ - and $y$-shifts between two speckle patterns can be determined simultaneously by performing a series of calculations for all relative $x-y$-positions of the two patterns involved, in a predetermined grid. This yields a crosscorrelation result: the $x-y$-position of the highest correlation coefficient, representing the $x$ - and $y$-shifts, and its value. A shift of one of the images corresponding to the $x-y$-position of the cross-correlation peak then gives the best alignment correction for repositioning the two images. However, this position may not yield the highest correlation: an additional sub-pixel shift may be required. Therefore a three-point, second degree polynomial fitting procedure involving only the highest value of the correlation coefficient and its two neighbours in either the $x$ - or the $y$-direction has been used to estimate the sub-pixel shift required for best match in both $x$ - and $y$-directions (figure 1(b)).

The estimated sub-pixel shift can be used within a nulling procedure based on a voltage controlled high resolution adjustment mechanism to align two images precisely.

Another error source that has to be corrected is relative rotation between two speckle patterns. Rotation can be caused by tilt errors in $45^{\circ}$ mirror surfaces that are present in the system, and can be corrected by an appropriate counteracting tilt of a $45^{\circ}$ mirror over its horizontal axis, as illustrated in figure 2. An image is reflected over an angle of $90^{\circ}$ by a $45^{\circ}$ mirror. If the mirror is positioned vertically the image is mirrored around a vertical axis. The mirrored image can be rotated in the observation plane by rotating the mirror around its horizontal axis. This rotation $\alpha$ can be decomposed in two components: $\alpha / \sqrt{2}$ in the $x$-direction, which is the optical axis of the incoming rays, and $\alpha / \sqrt{2}$ in the $y$-direction, corresponding to the optical axis of the reflected rays. Rotation of the mirror along the $x$-axis causes translation of the image at the observation plane; rotation along the $y$-axis causes rotation of the reflected image in the observation plane. If a mirror is adjusted over an angle of $\varphi$, reflected rays change over $2 \varphi$. Analogous to this, a rotation of $\alpha / \sqrt{2}$ of the $45^{\circ}$ mirror along the $y$-axis produces a rotation of the reflected image of $\alpha \sqrt{2}$. Rotations are indicated by double pointed arrows in figure 2 .

Rotation of an image causes its pixels to rotate around the centre of rotation. It can be seen easily that pixel shift caused

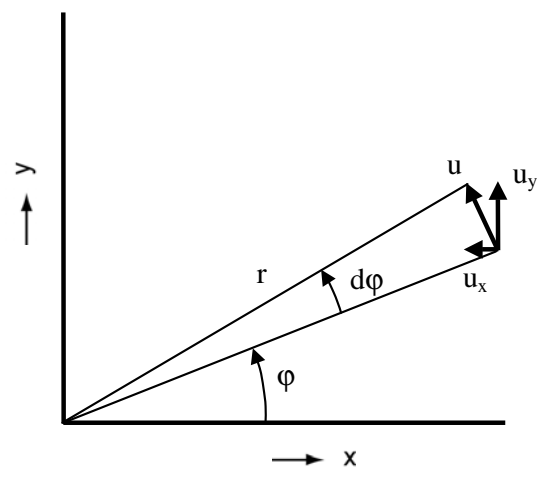

Figure 3. Pixel shifts $u_{x}$ and $u_{y}$ caused by rotation are proportional to $y$ - and $x$-coordinates respectively.

by rotation is constant for columns and rows, and proportional to the $x$ - or $y$-coordinate. Pixel shift $u$ caused by rotation of $\mathrm{d} \varphi$ is proportional to the radius, and is perpendicular to it (figure 3):

$$
u=r \mathrm{~d} \varphi .
$$

Pixel shift can be decomposed along the horizontal and vertical axes:

$$
\begin{gathered}
u_{x}=-u \sin \varphi \\
u_{y}=u \cos \varphi .
\end{gathered}
$$

Substituting equation (2) in equations (3) and (4) and converting $r$ to Cartesian coordinates yields

$$
\begin{gathered}
u_{x}=-y \mathrm{~d} \varphi \\
u_{y}=x \mathrm{~d} \varphi .
\end{gathered}
$$

The information required for adjusting the orientation of an image to achieve rotational alignment can be obtained by evaluating the correlation coefficient locally. Both images involved can be divided into a number of sub-images, and correlation can be calculated for all sub-images. The result of this is a $2 \mathrm{D}$ data set providing local information on the level of matching between two corresponding sub-images. The values of the local correlation coefficients can be plotted in a 2D map representing the local match between the two images. The highest value can be expected in the centre of rotation; correlation coefficients for sub-images at a certain distance from the rotation centre will be lower, and constant over a circle with its centre in the rotation centre. A tool based on this method can be helpful for the optimization of relative orientation of the two images.

Other, more powerful tools are based on the calculation of cross-correlation for all corresponding sub-images. This results in three $2 \mathrm{D}$ data sets, one for the peak values of the correlation coefficients of all sub-images, and two for local $x$ and $y$-positions of the cross-correlation peaks. The positions of cross-correlation peaks can be determined with sub-pixel resolution when using three-point parabola fitting as explained before. The data can be presented in different ways, for instance relative $x$ - and $y$-shifts of local cross-correlation peaks can be plotted as functions of $y$ - and $x$-position respectively. These plots are simple straight lines.

Since translation caused by rotation is constant for each row and column, the 2D data sets for local $x$ - and $y$-positions of 


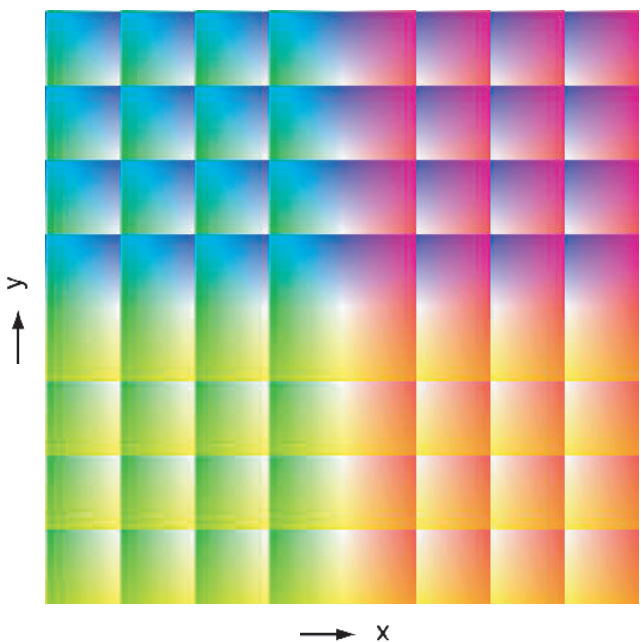

Figure 4. Colour coded 2D displacements caused by image rotation. White represents zero shift, blue (in grey-scale representation four horizontal dark grey fringes above the centre) and yellow (four horizontal light grey fringes below the centre) positive and negative $x$-shifts respectively. Green (four vertical medium grey fringes on the left) and red (four vertical dark grey fringes on the right) represent positive and negative $y$-shifts. Interpolated colours indicate combinations of $x$ - and $y$-shifts. The centre of rotation is in the centre of the image. Wrapping is applied at 0.25 pixel; $x$ - and $y$-shifts range from -1.0 to +1.0 pixels.

cross-correlation peaks can be averaged for rows and columns, respectively. This results in two sets of $1 \mathrm{D}$ data that can be used in a linear fitting procedure to obtain an error indication for rotation that is based on all available local cross-correlation peak shift data, reducing the influence of noise.

The $x$ - and $y$-displacements can be colour coded to present a 2D displacement field in one image. A colour scheme can be chosen such that zero shift is represented by the colour white. Green and red may represent positive and negative $y$-shifts of local cross-correlation peaks respectively; blue and yellow can represent positive and negative $x$-shifts. Combinations are obtained by bi-linear interpolation. To enhance visibility of small shifts the colour scheme can be wrapped, for instance at multiples of 0.25 pixel. As an example a $2 \mathrm{D}$ dataset representing a rotation with its centre in the origin is shown in figure 4. Rotation related $x$ - and $y$-translations are one-pixel at the edges. These shifts are converted by RGB colour look-up operations as described above.

\section{Implementation}

The described alignment methods have been implemented in a two-bucket one-camera shearing speckle interferometer (figure 5). This interferometer is the successor of a fourbucket two-camera speckle interferometer [9] that relied on mechanical stability to maintain its alignment, but still needed regular mechanical adjustment, and appeared to be very difficult to align. During the design of the current interferometer no special attention has been given to long term mechanical stability. Standard mirror holders and commercial lenses were used, with non-ideal thermal and mechanical properties. In order to compensate for these non-ideal characteristics a high resolution adjustment mechanism, based on a piezo-controlled mirror, has been incorporated into the design, providing sufficient short term stability for performing tests.

The interferometer has two optical branches, in one of which a phase step of $90^{\circ}$ is introduced. This branch starts at beam splitter $\mathrm{BC} 1$, and includes polarizing beam combiner $\mathrm{PBC} 2$, with phase stepper and quarter wave plate attached to it, and piezo-controlled mirror M4. P-polarized light leaving $\mathrm{PBC} 2$ towards mirror M4 is rotated through $90^{\circ}$ by the quarter wave plate and is then reflected by PBC2 towards the CCD. Mirror M4 is used for horizontal and vertical adjustment of the left image on the CCD. The other branch also starts at beam splitter $\mathrm{BC} 1$, and includes prism $\mathrm{P} 1,45^{\circ}$ mirror $\mathrm{M} 3$, and $\mathrm{PBC} 2$. Mirror M3 directs the light of this branch towards the $\mathrm{CCD}$, via PBC2. Mirror M3 is used for horizontal and vertical adjustment of the right image. Both images are projected onto the CCD by the same lens L3.

The interferometer has been designed in such a way that both images are projected symmetrically side by side onto a single CCD (figure 6)

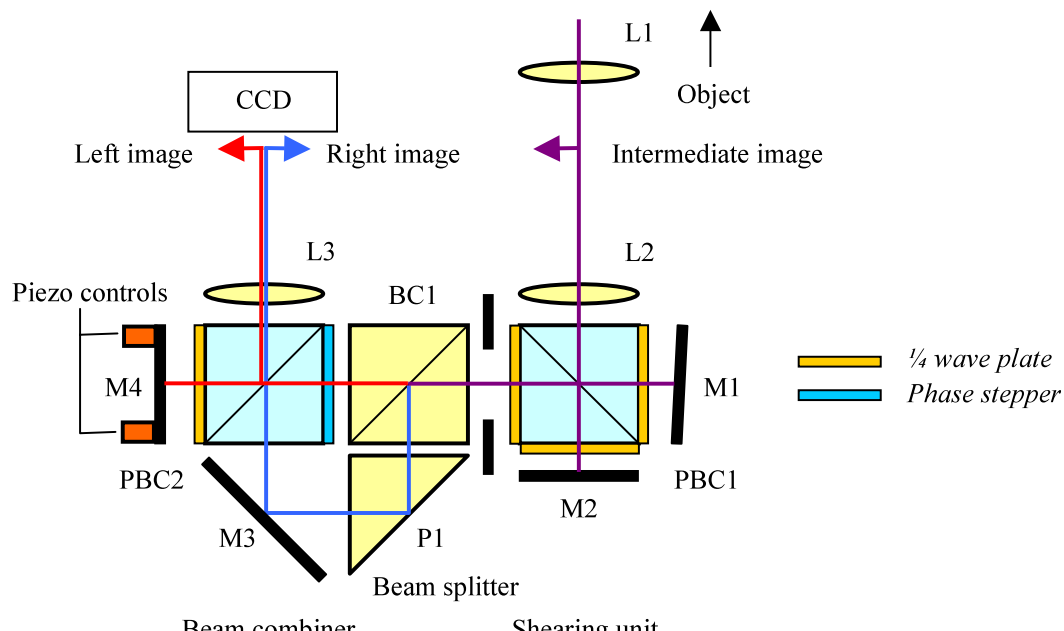

Figure 5. Two-bucket shearing speckle interferometer. 


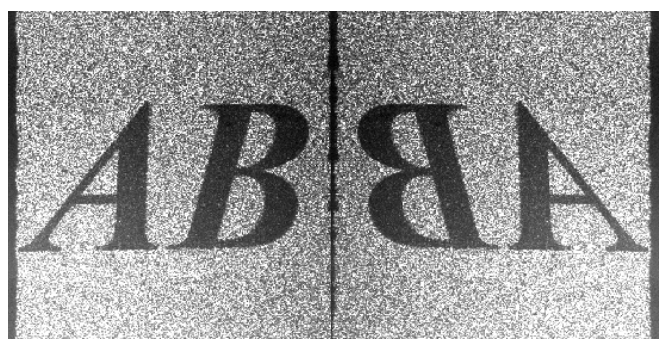

Figure 6. Symmetrically projected speckle patterns onto a single CCD.

The use of a single camera prevents problems that might arise from differences in focusing, magnification, and distortion, when two cameras with different imaging lenses are used. Symmetrical projection of the two images is achieved by the presence of an even number of mirrors in one optical branch, and an odd number in the other one. As a consequence, both images have the same level of symmetrical distortion caused by the imaging lens at corresponding pixel positions.

Alignment procedures have been developed for initial alignment, after assembling the interferometer, and for regular alignment, in order to maintain optimal settings during operation.

Initial alignment comprises the following:

- Alignment for the relative position of both images.

- Alignment for the relative orientation of the images.

Initial alignment of the relative position of both images takes place in three steps:

- Mechanical alignment to within three pixels.

- Software based course alignment to within one pixel.

- Sub-pixel alignment.

Regular alignment generally only involves the last two steps of position alignment.

Initial course alignment for relative position takes place by mechanical adjustment of mirrors M3 and M4 while blocking mirror M1 to prevent modulation, and after initial centring of mirror M2. Image subtraction of left and right image provides a reliable tool for course adjustment to better than three pixels. Although it is possible to perform this operation in speckled illumination, white light is preferred here.

During the next step in the initial alignment procedure for relative position, cross-correlation is calculated for the central areas $(64 \times 64$ pixels $)$ of both images, with a sufficiently large range of -16 to +16 pixels. The $x$ - and $y$-results indicate the pixel shifts that have to be introduced to achieve best match on pixel level, and are used when extracting both speckle patterns from the $1024 \times 1024$ CCD image to obtain pixel alignment.

The pixel-aligned images provide a starting point for subsequent sub-pixel adjustment by piezo-controlled mirror M4, based on three-point parabola fitting of cross-correlation results. The voltage for the piezo mirror is adjusted manually at present, but can be computer controlled for fully automated alignment. Calculation of cross-correlation and presentation of a cross-hair indicating actual sub-pixel image position is fast enough to allow for interactive adjustment of the mirror (figure 7).

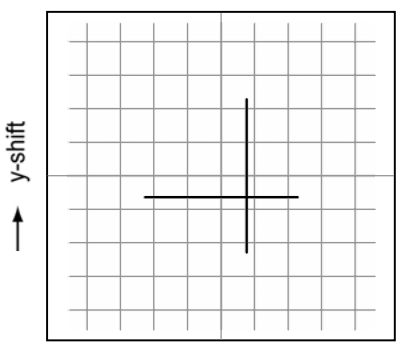

(a)

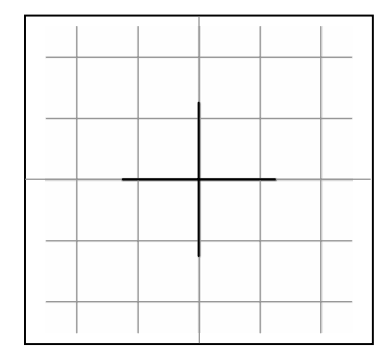

(b)
Figure 7. Real-time cross-hair indicators showing sub-pixel shift. Grid: 1 pixel. (a) Misalignment 0.8 pixels horizontally, 0.7 pixels vertically. (b) Perfect alignment.

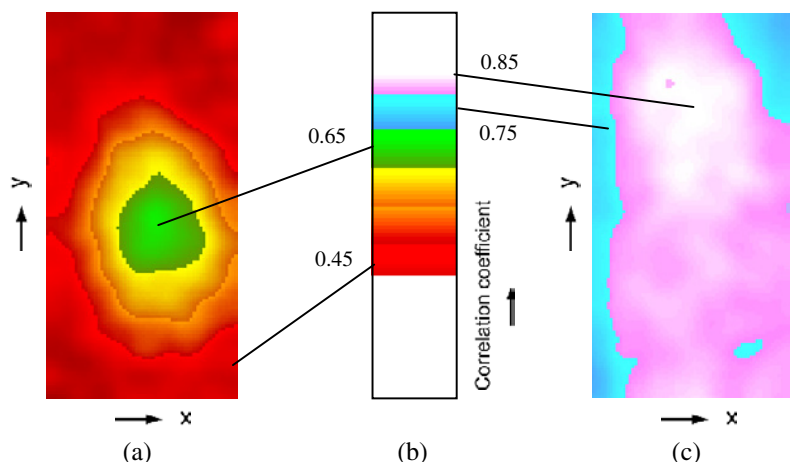

Figure 8. Local correlation of two speckle patterns. (a) Large relative rotation, local correlation coefficients ranging from 0.45 to 0.65. (b) Colour look-up bar graph. (c) No relative rotation, correlation 0.75 to 0.85 .

The other part of the initial alignment procedure is adjustment of relative orientation of both images. For this purpose $45^{\circ}$ mirror M3 is adjusted around its horizontal axis, providing a small amount of rotation of the image it projects onto the camera. Vertical translation of the image is followed by the other image by adjusting mirror M4 along its horizontal axis.

Relative rotation of the speckle patterns leads to decreasing local correlation coefficients for corresponding sub-images, with the highest value in the rotation centre, as described in section 2. A colour-coded 2D plot of local correlation coefficient values, given in figure 8 , shows this behaviour. In figure 8 (a) the local results for two $512 \times 1024$ images with large relative rotation are given. Local correlation coefficients were calculated on non-overlapping sub-images of $8 \times 8$ pixels. Figure 8 (b) shows a bar graph with the used section of the colour look-up table for the correlation coefficients. Figure 9(c) shows a colour-coded plot for zero rotation, showing a fairly uniform distribution of correlation coefficients over the image. The correlation coefficients range from 0.45 to 0.65 for the image showing large rotation, and from 0.75 to 0.85 for the one without rotation.

Shifts caused by rotation are constant over rows and columns, as described in section 2. This is illustrated by the 2D plots in figure 9, where cross-correlation results for all sub-images are plotted using the colour scheme as described in section 2. Again, local cross-correlation calculations were carried out on non-overlapping sub-images of $8 \times 8$ pixels. Figure 9(a) shows a distinct relative rotation between the two 


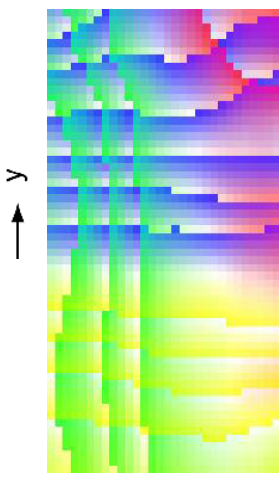

(a) $x$

(b)

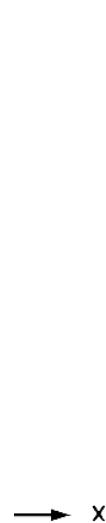

Figure 9. Local cross-correlation of two speckle patterns. (a) Relative rotation leading to positive horizontal shift of 1.5 pixels at the top and 1.0 negative shift at the bottom. (b) No relative rotation: residual shift values estimated smaller than 0.1 pixels, except at the top right.

patterns. Six horizontal wrappings are visible in blue (dark grey in grey-scale representation) above the centre, indicating a 1.5 pixel positive $x$-shift at the top of the image. Below the centre four yellow (light grey) wrappings are visible, indicating a 1.0 pixel negative $x$-shift at the bottom. At the same time three green (medium grey) wrappings are visible left of the centre, and one red (dark grey) wrapping at the top right. Figure 9(b), presenting local cross-correlation for two speckle patterns without relative rotation, shows no wrappings. A large region of the image has a colour close to white, indicating no $x$ - or $y$-shift, and except in the top right area no shift larger than 0.1 pixel is present.

When two images are perfectly aligned with respect to position and orientation, the $2 \mathrm{D}$ data sets representing the $x$ - and $y$-positions of local cross-correlation peaks should contain zero values (figure 9(b)). Any residual non-zero content indicates errors in the third category mentioned in section 1: differences in focusing, magnification and geometrical aberrations of the imaging optics for the two speckle patterns

Other helpful tools that were used during alignment of orientation were histograms and cluster plots (figure 10) Well aligned speckle patterns are characterized by narrow distributions of $x$ - and $y$-shifts of local cross-correlation peaks and a small cluster plot, showing $x$ - and $y$-distributions plotted against each other. With these tools $x$ - and $y$-shifts can also be observed easily.

Regular adjustment on the sub-pixel level, preceded by extraction of both images from appropriate positions in the raw image, as described above, only requires sub-pixel adjustment of piezo-driven mirror M4, and can be performed quickly. The piezo mirror used is able to maintain its position for a period of time that is relatively long compared with the duration of a test: 30-60 $\mathrm{min}$, compared to $1 \mathrm{~min}$ for a test. This procedure, without any mechanical adjustment, remains feasible as long as mechanical alignment stays within its predetermined limit of 16 pixels. For the case where mechanical adjustment of mirror M4 is required, its manual adjusters are accessible from outside the interferometer, so course alignment can take place without opening the casing. This has been necessary only on a few occasions. The software procedure that is used for regular alignment detects if image shift in the regular course alignment procedure exceeds the predetermined limit, and alerts the operator that mechanical adjustment of mirror M4 is necessary. The mechanical properties of the interferometer are appropriate to maintain relative orientation of the two images for a long period of time, so rotation alignment is only performed as part of initial adjustment.

As stated before, the images that are used for calculation of cross-correlation are not identical. For this reason mirror M1 has been blocked during initial alignment to prevent modulation, but even when modulation was present it was possible to perform sub-pixel alignment under laboratory conditions. Instead of blocking mirror M1, a horizontal or vertical polarizer can also be placed in front of field lens L1. Since our interferometer is polarization sensitive, only one polarization state after $\mathrm{PBC} 1$ remains when this polarizer is present, and no interference occurs, resulting in two identical speckle patterns on the camera.

\section{Experimental results}

An experiment that illustrates the importance of adequate alignment, and shows the effects of insufficient alignment, has been carried out. The test specimen used in this test was a Fokker 100 airbrake, consisting of a $0.3 \mathrm{~mm}$ aluminium skin, bonded on an aluminium honeycomb core. The specimen contained artificial defects of $24 \mathrm{~mm}$ diameter. The specimen was thermally loaded by an incandescent lamp for about one second, and relaxation of deformation was measured during cooling down. Data processing was performed for two

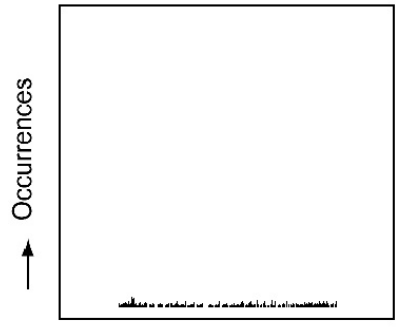

(a)

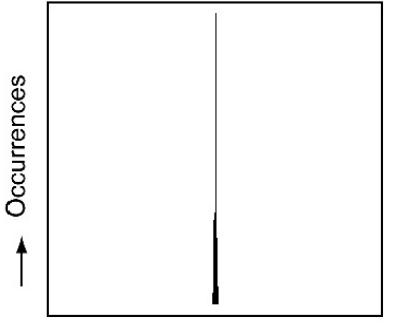

(b)

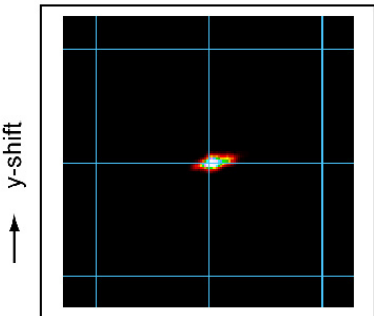

(c) $\longrightarrow$ x-shift

Figure 10. Histograms and cluster plots of $x$ - and $y$-shifts of local cross-correlation peaks. (a) Distribution of $x$-shifts for two speckle patterns with a rotation error, (b) the same distribution for well aligned patterns, (c) a cluster plot for well aligned patterns, $x$-shifts plotted against $y$-shifts. Grid: 1 pixel. 
Maintaining sub-pixel alignment for a single-camera two-bucket shearing speckle interferometer

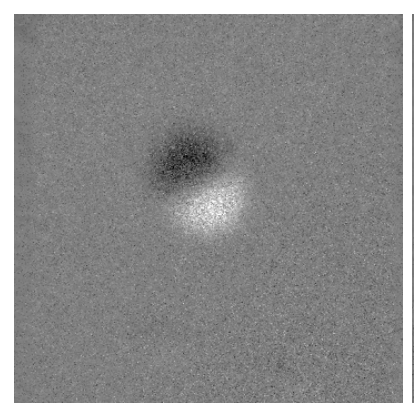

(a)

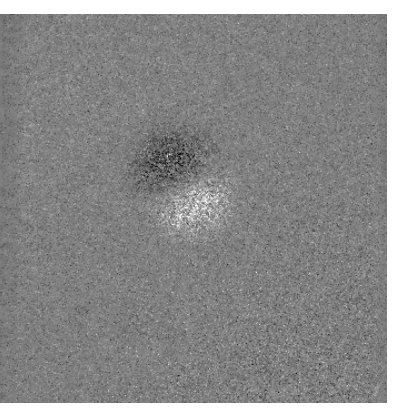

(b)

Figure 11. Defect indication in a Fokker 100 air brake. (a) Phase difference obtained with a well aligned system; (b) the same data, processed with 1 pixel misalignment between the two phase stepped speckle patterns.

alignment settings: optimum setting, and 1 pixel horizontal misalignment. Figure 11 shows the results.

\section{Conclusions}

Precise alignment is crucial for multi-channel phase stepped speckle interferometers. Speckle patterns can be aligned for position and orientation using tools that are based on global and local correlation and cross-correlation. Current computer technology allows interactive alignment. Long term stability, which usually relies on mechanical stability, is not a primary requirement any more for this type of interferometer, thanks to the incorporation in the alignment system of a piezo-controlled mirror with sub-pixel resolution.

\section{Acknowledgments}

This research was supported by the Technology Foundation STW, the Applied Science Division of NWO and the Technology Programme of the Ministry of Economic Affairs.

\section{References}

[1] Creath K 1988 Phase-measurement interferometry techniques Progress in Optics vol 26, ed E Wolf (Amsterdam: Elsevier) pp 349-93

[2] Kujawinska M 1993 Spatial phase measurement methods Interferogram Analysis-Digital Fringe Pattern Measurement Techniques ed D W Robinson and G T Reid (Bristol: Institute of Physics Publishing) chapter 5, pp 141-93

[3] Bothe T, Burke J and Helmers H 1997 Spatial phase shifting in ESPI: minimization of phase reconstruction errors Appl. Opt. 36 5310-6

[4] Kwon O Y 1984 Multichannel phase-shifted interferometer Opt. Lett. 9 59-61

[5] Kujawinska M and Robinson D W 1988 Multichannel phase-stepped holographic interferometry Appl. Opt. 27 312-20

[6] Schwider J 1996 Echtzeitinterferometer mit 'phase-shift-auswertung' in 'single frame'-technik Patent Specification DE19652113.0

[7] Barrientos B, Moore A J, Perez-Lopez C, Wang L L and Tschudi T 1997 Transient deformation measurement with ESPI using a diffractive optical element for spatial phase-stepping Automatic Processing of Fringe Patterns ed W Jüptner and W Osten (Berlin: Akademie) pp 371-5

[8] van Haasteren A J P and Frankena H J 1994 Real-time displacement measurement using a multicamera phase-stepping speckle interferometer Appl. Opt. 33 $4137-42$

[9] Weijers A L, van Brug H and Frankena H J 1998 Polarization phase stepping with a Savart element Appl. Opt. 37 5150-5

[10] Somers P A A M and van Brug H 2001 A single camera, dual image real-time-phase-stepped shearing speckle interferometer Proc. Fringe 2001 ed W Osten and W Jüptner (Amsterdam: Elsevier) pp 573-80

[11] Goodman J W 1975 Statistical properties of laser speckle patterns Topics in Applied Physics vol 9 Laser Speckle and Related Phenomena ed J C Dainty (Berlin: Springer)

[12] Yamaguchi I 1981 Speckle displacement and decorrelation in the diffraction and image fields for small object deformation Opt. Acta 28 1359-76

[13] Sjödahl M and Benckert L R 1993 Electronic speckle photography: analysis of an algorithm giving the displacement with subpixel accuracy Appl. Opt. 32 2278-84 